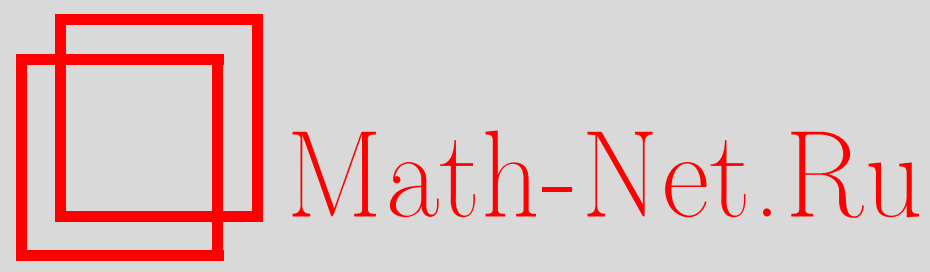

О. В. Шемякина, О перемешивающих свойствах операций в конечном поле, Дискрет. матем., 2011, том 23, выпуск $2,32-40$

DOI: https://doi.org/10.4213/dm1138

Использование Общероссийского математического портала Math-Net.Ru подразумевает, что вы прочитали и согласны с пользовательским соглашением http://www . mathnet.ru/rus/agreement

Параметры загрузки:

IP : 54.224 .60 .19

26 апреля 2023 г., 15:49:31 
УДК 519.7

\title{
О перемешивающих свойствах операций в конечном поле
}

\author{
(c) 2011 г. О. В. Шемякина
}

\begin{abstract}
$\mathrm{C}$ точки зрения подхода, изложенного в работе Шеннона [1], представляют интерес перемешивающие свойства операций в конечном поле, в частности, действие каждой операции на смежные классы относительно второй операции. Хорошие перемешивающие свойства операций позволяют строить на их основе стойкие криптографические алгоритмы.
\end{abstract}

\section{1. Введение}

Операции в конечном поле легко реализуются на современных вычислительных устройствах. Поэтому эти операции являются перспективными для построения различных криптографических алгоритмов. С точки зрения подхода, изложенного в работе Шеннона [1], представляют интерес перемешивающие свойства данных операций. Сложение сохраняет смежные классы аддитивной группы поля по любой аддитивной подгруппе. Умножение сохраняет смежные классы мультипликативной группы поля по любой мультипликативной подгруппе. Это позволяет проводить криптографический анализ методом гомоморфизмов $[2,3]$.

В работе рассматривается действие каждой из операций в конечном поле $G F\left(q^{n}\right)$, где $q-$ степень простого числа, $n>1$, на смежные классы относительно второй операции. Хорошие перемешивающие свойства обеспечивают стойкость композиции двух операций к криптоанализу на основе гомоморфизмов и позволяют проектировать итеративные шифры с использованием операций в конечном поле.

\section{2. Действие операции сложения на смежные классы по мультипликативной группе подполя}

Рассмотрим действие операции сложения в поле $G F\left(q^{n}\right)$ на элементы факторгруппы $G F\left(q^{N}\right)^{*} / G F(q)^{*}$. Представляет интерес распределение по смежным классам элементов суммы двух фиксированных смежных классов. Поскольку мультипликативная группа конечного поля и любая ее подгруппа являются циклическими, факторгруппа $G F\left(q^{N}\right)^{*} / G F(q)^{*}$ изоморфна циклической группе $G F\left(q^{N}\right)^{*} / G F(q)^{*} \simeq\langle\beta\rangle$.

Обозначим $\left[\beta^{i}\right], 0 \leqslant i<\left(q^{n}-1\right) /(q-1)$, смежный класс, порожденный элементом $\beta^{i}:\left[\beta^{i}\right] \in G F\left(q^{n}\right)^{*} / G F(q)^{*}$. Под суммой смежных классов $\left[\beta^{h}\right]$ и $\left[\beta^{t}\right]$, где $\left[\beta^{h}\right],\left[\beta^{t}\right] \in$ $G F\left(q^{N}\right)^{*} / G F(q)^{*}$, понимается множество

$$
\left[\beta^{h}\right]+\left[\beta^{t}\right]=\left\{x+y \mid x \in\left[\beta^{h}\right], y \in\left[\beta^{t}\right]\right\} .
$$


Лемма 1. При $\left[\beta^{h}\right],\left[\beta^{t}\right] \in G F\left(q^{n}\right)^{*} / G F(q)^{*},\left[\beta^{h}\right] \neq\left[\beta^{t}\right]$, элементы множества $\left[\beta^{h}\right]+\left[\beta^{t}\right]$ различны, не равны нулю и не попадают в классы $\left[\beta^{h}\right] u\left[\beta^{t}\right]$.

Доказательство. Пусть не все элементы различны и для некоторых $x_{1}, x_{2}, y_{1}, y_{2}$, принадлежащих $G F(q)^{*}$, где, не умаляя общности, $x_{1} \neq y_{1}$, справедливо равенство

$$
\beta^{h} x_{1}+\beta^{y} x_{2}=\beta^{h} y_{1}+\beta_{t} y_{2} .
$$

Поскольку множество элементов $G F(q)$ * замкнуто относительно сложения, справедливо включение $\beta^{h-t}=\left(y_{2}-x_{2}\right) y_{1}-x_{1}{ }^{-1} \in G F(q)^{*}$, что возможно только при $h=t$.

Предположим теперь, что для $x_{1}, x_{2} \in G F(q) *$ справедливо равенство

$$
\beta^{h} x_{1}+\beta^{t} x+2=0 \text {. }
$$

Тогда $\beta^{h-t}=-x_{2} x_{1}^{-1} \in G F(q)$, что опять возможно лишь при $h=t$.

Покажем, что элементы множества $\left[\beta^{h}\right]+\left[\beta^{t}\right]$ не попадают в класс $\left[\beta^{h}\right]$. Пусть это не так и для некоторых $x_{1}, x_{2}, x_{3} \in G F(q)^{*}$ справедливо равенство

$$
\beta^{h} x_{1}+\beta^{t} x_{2}=\beta^{h} x_{3} .
$$

Тогда $x_{1} \neq x_{3}$ и $\beta^{h-t}=x_{2}\left(z_{3}-x_{1}\right)^{-1}$, что, как показано выше, влечет выполнение условия $h=t$.

Аналогично, элементы множества $\left[\beta^{h}\right]+\left[\beta^{t}\right]$ не попадают в класс $\left.\beta^{t}\right]$.

Лемма 2. Пусть $H$ - произвольная подгруппа группь $G F\left(q^{n}\right)^{*}$ Если для смежных классов $\left[\beta^{h}\right],\left[\beta^{t}\right] \in G F\left(q^{n}\right)^{*} / H$ множество $\left[\beta^{h}\right]+\left[\beta^{t}\right]$ содержит элемент некоторого смежного класса $\left[\beta^{s}\right] \in G F\left(q^{n}\right)^{*}$, то оно содержит все элементы этого класса.

Доказательство. Группа $H$ является циклической. Обозначим $\alpha$ ее образующую. Пусть для некоторых $x_{1}, x_{2}, x_{3} \in H$ справедливо равенство

$$
\beta^{h} x_{1}+\beta^{t} x_{2}=\beta^{s} x_{3} .
$$

Тогда

$$
\beta^{h} x_{1} \alpha^{i}+\beta^{t} x_{2} \alpha^{i}=\beta^{s} x_{3} \alpha^{i}
$$

для всех $i=0,1, \ldots,|H|-1$. Значения в правой части различны при разных значениях $i$ и образуют смежный класс $\left[\beta^{s}\right]$.

Предложение 1. (1) Каждый элемент суммы $\left[\beta^{h}\right]+\left[\beta^{t}\right]$ для смежного класса $\left[\beta^{h}\right] \in G F\left(q^{n}\right)^{*} / G F(q)^{*}$ либо равен нулю, либо принадлежит $\left[\beta^{h}\right]$. При этом нулевой элемент встречается в этой сумме $s-1$ раз, каждый ненулевой элемент $x \in\left[\beta^{h}\right]$ встречается $q-2$ раза.

(2) Все элементы суммы $\left[\beta^{h}\right]+\left[\beta^{t}\right]$, где $\left[\beta^{h}\right],\left[\beta^{t}\right] \in G F\left(q^{n}\right)^{*} / G F(q)^{*} u\left[\beta^{h}\right] \neq\left[\beta^{t}\right]$, различны и составляют объединение всех элементов q-1 различных классов, отличных om $\left[\beta^{h}\right] u\left[\beta^{t}\right]$.

Доказательство. Значение в правой части равенства $\beta^{h} x_{1}+\beta^{h} x_{2}=\beta^{h}\left(x_{1}+x_{2}\right)$, где $x_{1}, x_{2} \in G F(q)^{*}$, равно нулю при $x_{2}=-x_{1}$. В остальных случаях оно принадлежит смежному классу $\left[\beta^{h}\right]$, причем по лемме 2 каждый элемент $\left[\beta^{h}\right]$ встретится в сумме $\left[\beta^{h}\right]+\left[\beta^{h}\right]$ одинаковое число раз. Это доказывает первое утверждение.

Второе утверждение непосредственно следует из лемм 1 и 2.

Следствие 1. В случае квадратичного расширения суммы $\left[\beta^{h}\right]+\left[\beta^{t}\right]$, где $\left[\beta^{h}\right],\left[\beta^{t}\right] \in$ $G F\left(q^{2}\right)^{*} / G F(q)^{*} u\left[\beta^{h}\right] \neq\left[\beta^{t}\right]$, является объединением всех отличных от $\left[\beta^{h}\right] u\left[\beta^{t}\right]$ смежных классов.

2 Дискретная математика, т.23 №2 


\section{3. Действие операции сложения на смежные классы по другим мультипликативным подгруппам}

Во втором разделе рассмотрена факторгруппа мультипликативной группы поля по мультипликативной группе подполя. Существуют факторгруппы мультипликативной группы поля по мультипликативной подгруппе, не являющейся мультипликативной группой подполя. Для таких факторгрупп в общем случае распределение элементов суммы двух смежных классов не является равномерным. Рассмотрим один частный случай - смежные классы мультипликативной группы поля $G F\left(q^{2}\right)^{*}$ по подгруппе порядка $q+1$. Этот случай является важным, поскольку для максимальной эффективности метода гомоморфизмов необходимо выбирать подгруппу мощности, близкой к величине квадратного корня из числа элементов в исходной группе. Пусть $H=\langle\beta\rangle-$ подгруппа $G F\left(q^{2}\right)^{*}$ порядка $q+1$. Тогда $\beta=\gamma^{q-1}$, где $\gamma-$ примитивный элемент поля $G F\left(q^{2}\right)$. Факторгруппа $G F\left(q^{2}\right)^{*} / H$ является циклической группой порядка $q-1$. Обозначим через $\left[\gamma^{i}\right], 0 \leqslant i \leqslant q-1$, смежный класс, порожденный элементом $\gamma^{i}:\left[\gamma^{i}\right]=\gamma^{i} H$. Представляет интерес распределение по смежным классам элементов суммы двух фиксированных смежных классов.

Лемма 3. Два ненулевых элемента $x_{1}$ и $x_{2}$ поля $G F\left(q^{2}\right)$ принадлежат одному смежному классу из $G F\left(q^{2}\right)^{*} / H$ тогда и только тогда, когда $x_{1}^{q+1}=x_{2}^{q+1}$.

Доказательство. Элементы $x_{1}$ и $x_{2}$ принадлежат одному смежному классу из $G F\left(q^{2}\right)^{*}$ тогда и только тогда, когда существует $h \in H: x_{2}=x_{1} h$, а это эквивалентно условию $x_{1}^{q+1}=x_{2}^{q+1}$, поскольку элементы $H$ и только они являются корнями из 1 степени $q+1$ в поле $G F\left(q^{2}\right)$.

Лемма 4. Если элементы $x_{1}$ и $x_{2}$ поля $G F\left(q^{2}\right)$ принадлежат различным смежным классам из $G F\left(q^{2}\right)^{*} / H$, по $x_{1}+x_{2} \neq 0$.

Доказательство. В случае четного $q$ для любого $x \in G F\left(q^{2}\right)$ справедливо равенство $-x=x$. Если $q$ является нечетным, то $-1=\beta^{(q+1) / 2}$, поскольку порядок $\beta$ равен $q+1$ и $-x=x \beta^{(q+1) / 2}$ для любого $x \in G F\left(q^{2}\right)$. Это означает, что противоположные элементы всегда принадлежат одному смежному классу.

Предложение 2. (1) При нечетном $q$ и нечетной сумме $i+j$ элементы суммы $\left[\gamma^{i}\right]+\left[\gamma^{j}\right]$ попадают в $(q+1) / 2$ смежных класса, при этом в каждый из этих классов попадает $2(q+1)$ элементов суммы.

(2) При нечетном $q$ и четной сумме $i+j$, где $i \neq j$, элементы суммы $\left[\gamma_{i}\right]+\left[\gamma^{j}\right]$ попадают в $(q+3) / 2$ смежных класса, при этом в два класса попадает по $q+1$ элементов суммы, в каждый из остальных - по $2(q+1)$ элементов суммы.

(3) При нечетном $q$ среди элементов суммы $\left[\gamma^{i}\right]+\left[\gamma^{i}\right]$ содержится $q+1$ нулевых элементов, остальные элементы попадают в $(q+1) / 2$ смежных класса, при этом в один класс попадает $q+1$ элемент суммы, в каждый из остальных - по $2(q+1)$ элементов суммы.

(4) При четном $q$ и условии $i \neq j$ элементы суммы $\left[\gamma_{i}\right]+\left[\gamma^{j}\right]$ попадают в $q / 2+1$ смежный класс, при этом в один класс попадает $q+1$ элемент суммь, в каждый из остальных - по $2(q+1)$ элементов суммы. 
(5) При четном q среди элементов суммы $\left[\gamma^{i}\right]+\left[\gamma^{i}\right]$ содержится $q+1$ нулевой элемент, остальные элементы попадают в q/2 смежных класса, при этом в каждый из этих классов попадает по $2(q+1)$ элементов суммыл.

Доказательство. Можно считать, что $i \leqslant j$. Достаточно доказать утверждение для случая $i=0$, поскольку

$$
\gamma^{i} H+\gamma^{j} H=\gamma^{i}(H+\gamma j-i H),
$$

и распределение этого множества по смежным классам отличается от распределения множества $H+\gamma^{j-i} H$ только перестановкой классов. Далее рассматриваем суммы

$$
H+\gamma^{s} H, \quad s \in\{0,1, \ldots, q-2\} .
$$

Из доказательства леммы 2 следует, что достаточно рассмотреть распределение по смежным классам элементов суммы (1) при фиксированном слагаемом из множества $H$.

В условиях первой части $s=2 t+1, t \in\{0,1, \ldots,(q-3) / 2\}$. Рассмотрим распределение по смежным классам сумм $\beta^{q-t}+\gamma^{2 t+1} \beta^{k}$, где $k \in\{0,1, \ldots, q\}$. Пр лемме 4 они ненулевые. Покажем, что $\beta^{q-t}+\gamma^{2 t+1} \beta^{k}$ и $\beta^{q-t}+\gamma^{2 t+1} \beta^{l}$, где $k, l \in\{0,1, \ldots, q\}, k<l$, принадлежат одному смежному классу тогда и только тогда, когда $l=q-k$. С учетом леммы 3 необходимо доказать, что

$$
\left(\beta^{q-t}+\gamma^{2 t+1} \beta^{k}\right)^{q+1}=\left(\beta^{q-t}+\gamma^{2 t+1} \beta^{l}\right)^{q+1} .
$$

Поскольку $q$ является степенью характеристики поля $G F\left(q^{2}\right)$,

$$
\begin{aligned}
\left(\beta^{q-t}+\gamma^{2 t+1} \beta^{k}\right)^{q+1}= & \left(\beta^{q-t}+\gamma^{2 t+1} \beta^{k}\right)^{q}\left(\beta^{q-t}+\gamma^{2 t+1} \beta^{k}\right) \\
= & \left(\beta^{(q-t) q}+\gamma^{(2 t+1) q} \beta^{k q}\right)\left(\beta^{q-t}+\gamma^{2 t+1} \beta^{k}\right) \\
= & \beta^{(q-t)(q+1)}+\gamma^{2 t+1} \beta^{(q-t) q+k}+\gamma^{(2 t+1) q} \beta^{k q+q-t} \\
& \quad+\gamma^{(2 t+1)(q+1)} \beta^{k(q+1)} .
\end{aligned}
$$

С учетом условия $\beta^{q+1}=1$ получаем, что равенство

$$
\left(\beta^{q-t}+\gamma^{2 t+1} \beta^{k}\right)^{q+1}=\left(\beta^{q-t}+\gamma^{2 t+1} \beta^{l}\right)^{q+1}
$$

эквивалентно равенству

$$
\gamma^{2 t+1} \beta^{(q-t) q+k}+\gamma^{(2 t+1) q} \beta^{k q+q-t}=\gamma^{2 q+1} \beta^{(q-t) q+l}+\gamma^{(2 t+1) q} \beta^{l q+q-t} .
$$

В силу соотношений $\gamma^{q}=\gamma \beta, \beta^{q^{2}}=\beta$ и $\beta^{q}=\beta^{-1}$, последнее эквивалентно равенству

$$
\gamma^{2 t+1} \beta^{1+t+k}+\gamma^{2 t+1} \beta^{2 t+1} \beta^{-k+q-t}=\gamma^{2 t+1} \beta^{1+t+l} \beta^{2 t+1} \beta^{-l+q-t},
$$

или, после сокращения на $\gamma^{2 t+1} \beta^{t+1}$, равенству

$$
\beta^{k}+\beta^{q-k}=\beta^{l}+\beta^{q-l}
$$

то есть,

$$
\beta^{l}\left(\beta^{k-l}-1\right)=\beta^{q-k}\left(\beta^{k-l}-1\right) .
$$

Так как $k, l \in\{0,1, \ldots, q, k<l$, это означает, что $l=q-k$. Поскольку $q$ нечетно, из показанного вытекает, что элементы суммы (1) при фиксированном слагаемом из класса $H$ принимают по 2 значения из $(q+1) / 2$ смежных классов. 
В условиях второй части $s=2 t+2, t \in\{0,1, \ldots,(q-5) / 2\}$. Доказательство аналогично доказательству первой части. Покажем, что суммы $\beta^{(q-1) / 2-t}+\gamma^{2 t+2} \beta^{k}$ и $\beta^{(q-1) / 2-t}+$ $\gamma^{2 t+2} \beta^{l}$, где $k, l \in\{1, \ldots, q\}, k<l$, принадлежат одному смежному классу тогда и только тогда, когда $l=q+1-k$. Равенство

$$
\left(\beta^{(q-1) / 2-t}+\gamma^{2 t+2} \beta^{k}\right)^{q+1}=\left(\beta^{(q-1) / 2-t}+\gamma^{2 t+2} \beta^{l}\right)^{q+1}
$$

эквивалентно равенству

$$
\begin{aligned}
\gamma^{2 t+2} \beta^{((q-1) / 2-t) q+k}+\gamma^{(2 t+2) q} \beta^{k q+(q-1) / 2-t} & \\
& =\gamma^{2 t+2} \beta^{((q-1) / 2-t) q+l}+\gamma^{(2 t+2) q} \beta^{k q+(q-1) / 2-t} .
\end{aligned}
$$

Воспользовавшись формулами для $\gamma^{q}$ и $\beta^{q}$ и сократив на $\gamma^{2 t+2} \beta^{t-(q-1) / 2}$, получаем равенство

$$
\beta^{k}+\beta^{q+1-k}=\beta^{l}+\beta^{q+1-l} .
$$

Поскольку $k \neq l$, равенство

$$
\beta^{l}\left(\beta^{k-l}-1\right)=\beta^{q+1-k}\left(\beta^{k-l}-1\right)
$$

эквивалентно условию $l=q+1-k$.

Осталось разобрать случай $k=0$ (при котором $q+1-k>0)$ и $k=(q+1) / 2$ (при котором $q+1-k=k)$. Покажем, что $\beta^{(q-1) / 2-t}+\gamma^{2 t+2}$ и $\beta^{(q-1) / 2-t}+\gamma^{2 t+2} \beta^{(q+1) / 2}$ принадлежат разным смежным классам. Пропуская несколько равносильных преобразований, которые полностью совпадают с написанными выше, получаем, что

$$
\left(\beta^{(q-1) / 2-t}+\gamma^{2 t+2}\right)^{q+1}=\left(\beta^{(q-1) / 2-t}+\gamma^{2 t+2} \beta^{(q+1) / 2}\right)^{q+1}
$$

выполняется тогда и только тогда, когда $1+\beta^{q+1}=\beta^{(q+1) / 2}+\beta^{q+1-(q+1) / 2}$, что эквивалентно условию $\beta^{(q+1) / 2}=1$, которое, очевидно, не выполняется, поскольку порядок $\beta$ равен $q+1$.

Для доказательства третьей части рассмотрим суммы $1+\beta^{k}$. При $k=(q+1) / 2$ и только в этом случае $1+\beta^{k}=0$. Покажем, что суммы $1+\beta^{k}$ и $1+\beta^{l}$, где $k, l \in\{1, \ldots, q\}$, $k<l$, принадлежат одному смежному классу тогда и только тогда, когда $l=q+1-k$. При условии $k<l$ рассматриваемые суммы ненулевые. Равенство $\left(1+\beta^{k}\right)^{q+1}=\left(1+\beta^{l}\right)^{q+1}$ сводится к равенству $\beta^{-k}+\beta^{k}=\beta^{l}+\beta^{-l}$. Воспользовавшись соотношением $\beta^{q+1}=1$, получаем, что $\beta^{q+1-k}+\beta^{k}=\beta^{q+1-l}+\beta^{l}$. Как уже было показано в ходе доказательства второй части, последнее эквивалентно равенству $l=q+1-k$.

Осталось рассмотреть случай $k=0$. Сумма $1+\beta^{k}=2$ попадает в некоторый смежный класс, который не может совпадать ни с одним из $(q-1) / 2$ смежных классов, содержащих суммы при $k \neq 0,(q+1) / 2$.

Доказательство четвертой части при нечетном $s=2 t+1, t \in\{0,1, \ldots, q / 2-1\}$ повторяет доказательство первой части. Суммы $\beta^{q-t}+\gamma^{2 t+1} \beta^{k}$ и $\beta^{q-t}+\gamma^{2 t+1} \beta^{l}$, где $k, l \in\{0,1, \ldots, q\}, k<l$, принадлежат одному смежному классу тогда и только тогда, когда $l=q-k$. Отличие состоит в том, что при четном $q$ условие $k<l$ не выполняется для $k=q / 2$. Поэтому элементы соответствующего класса встречаются в сумме (1) по одному разу. В случае четного $s=2 t+2, t \in\{0,1, \ldots, q / 2-2\}$, покажем, что суммы $\beta^{q / 2-1-t}+\gamma^{2 t+2} \beta^{k}$ и $\beta^{q / 2-1-t}+\gamma^{2 t+2} \beta^{l}$, где $k, l \in\{0,1, \ldots, q\}, k<l$, принадлежат одному смежному классу тогда и только тогда, когда $l=q-k$. Равенство

$$
\left(\beta^{q / 2-1-t}+\gamma^{2 t+2} \beta^{k}\right)^{q+1}=\left(\beta^{q / 2-1-t}+\gamma^{2 t+2} \beta^{l}\right)^{q+1}
$$


использованными выше способами сводится к равенству

$$
\beta^{t+1-q / 2+k}+\beta^{t+1+q / 2-k}=\beta^{t+1-q / 2+l}+\beta^{t+1+q / 2-l},
$$

которое после сокращения на $\beta^{t+1-q / 2}$ приводит к рассмотренному в первой части равенству $\beta^{k}+\beta^{q-k}=\beta^{l}+\beta^{q-l}$.

Как и в случае нечетного $s$, элементы смежного класса, содержащего элемент $\beta^{q / 2-1-t}+\gamma^{2 t+2} \beta^{q / 2}$, встречаются в сумме (1) по одному разу.

Для доказательства пятой части рассмотрим суммы $\beta^{q / 2}+\beta^{k}$. При $k=q / 2$ и только в этом случае $\beta^{q / 2}+\beta^{k}=0$. Можно показать, что $\beta^{q / 2}+\beta^{k}$ и $\beta^{q / 2}+\beta^{l}$, где $k, l \in$ $\{0,1, \ldots, q\}, k<l$, принадлежат одному смежному классу тогда и только тогда, когда $l=q-k$. Стандартными преобразованиями равенство $\left(\beta^{q / 2}+\beta^{k}\right)^{q+1}=\left(\beta^{q / 2}+\beta^{l}\right)^{q+1}$ сводится к равенству $\beta^{k}+\beta^{q-k}=\beta^{l}+\beta^{q-l}$.

\section{4. Действие операции умножения на смежные классы по аддитивной группе подполя}

Рассмотрим действие операции умножения в поле $G F\left(q^{2}\right)$ на элементы факторгруппы $G F\left(q^{n}\right) / G F(q)$. Представляет интерес распределение по смежным классам элементов произведения двух фиксированных смежных классов. Под произведением смежных классов $[h]$ и $[t]$, где $[h],[t]$ принадлежат $G F\left(q^{n}\right) / G F(q)$, понимается множество

$$
[h] \cdot[t]=\{x y \mid x \in[h], y \in[t]\} .
$$

Нулевым смежным классом [0] является $G F(q)$.

Предложение 3. (1) Все элементы произведения [0]·[0] принадлежат смежному классу [0]. При этом каждый ненулевой элемент $G F(q)$ встречается в этом произведении $q-1$ раз, нулевой элемент встречается $2 q-1$ раз.

(2) Произведение двух смежных классов из $G F\left(q^{n}\right) / G F(q)$, по крайней мере один из которых не является нулевым, содержит либо по q смежных классов (не обязательно различных) из q различных смежных классов, либо по одному элементу из $q^{2}$ различных смежных классов.

Доказательство. Смежный класс [0] является полем $G F(q)$, поэтому произведение любых двух элементов из [0] принадлежит этому же смежному классу. Произведение двух элементов поля равно нулю тогда и только тогда, когда по крайней мере один из сомножителей нулевой, поэтому нулевой элемент встречается $2 q-1$ раз. Если один ненулевой сомножитель зафиксирован, а второй принимает все значения из $G F(q)^{*}$, то и произведение принимает все значения из $G F(q)^{*}$. Первое утверждение доказано.

Пусть $\left\{1, \alpha, \alpha^{2}, \ldots, \alpha^{n-1}\right\}-$ базис $G F\left(q^{n}\right)$ как векторного пространства над $G F(q)$. Тогда $[s] \in G F\left(q^{n}\right) / G F(q)$ можно представить в виде суммы

$$
[s]=s+G F(q)=\sum_{i=1}^{n-1} s_{i} \alpha^{i}+G F(q),
$$

где $s_{i} \in G F(q)$. Пусть $[h] \neq[0]$. Рассмотрим произведение

$$
[h] \cdot[t]=\left(\sum_{i=1}^{n-1} h_{i} \alpha^{i}+G F(q)\right)\left(\sum_{i=1}^{n-1} t_{i} \alpha^{i}+G F(q)\right) .
$$


Сначала докажем, что если некоторый смежный класс $[s]$ содержит два элемента произведения $[h] \cdot[t]$, то он содержит по крайней мере $q$ элементов этого произведения. Пусть для $x_{1}, x_{2}, y_{1}, y_{2} \in G F(q)$, где равенства $x_{1}=x+2$ и $y_{1}=y_{2}$ не выполняются одновременно, произведения $\left(h+x_{1}\right)\left(t+y_{1}\right)$ и $\left(h+x_{2}\right)\left(t+y_{2}\right)$ принадлежат одному смежному классу, то есть

$$
\left(h+x_{1}\right)\left(t+y_{1}\right)-\left(h+x_{2}\right)\left(t+y_{2}\right) \in G F(q) .
$$

Это означает, что

$$
\sum_{i=1}^{n-1}\left(h_{i} y_{1}+t_{i} x_{1}\right) \alpha^{i}=\sum_{i=1}^{n-1}\left(h_{i} y_{2}+t_{i} x_{2}\right) \alpha^{i} .
$$

Поскольку степени элемента $\alpha_{i}$ являются линейно независимыми, условие (2) эквивалентно выполнению равенства

$$
h_{i} y_{1}+t_{i} x_{1}=h_{i} y_{2}+t_{i} x_{2}
$$

для всех $i=1, \ldots, n-1$. Из (2) и условия $[h] \neq[0]$ следует, что $x_{1} \neq x_{2}$. Равенства (3) для всех $i=1, \ldots, n-1$, для которых $h_{i} \neq 0$, эквивалентны условиям

$$
\frac{t_{i}}{h_{i}}=\frac{y_{2}-y_{1}}{x_{1}-x_{2}}
$$

Значение в правой части (4) принадлежит полю $G F(q)$ и не зависит от $i$. Обозначим его $c$. Тогда для любого элемента $x^{\prime} \in G F(q)$ и $y^{\prime}=c\left(x_{1}-x^{\prime}\right)+y_{1}$, также принадлежащего $G F(q)$ для всех $i=1, \ldots, n-1$, для которых $h_{i} \neq 0$, будут выполняться равенства

$$
h_{i} y_{1}+t_{i} x_{1}=h_{i} y^{\prime}+t_{i} x^{\prime}
$$

Если $h_{i}=0$, то из (3) и условия $x_{1} \neq x_{2}$ следует, что $t_{i} \neq 0$. Таким образом, равенства (5) выполняются для всех $i=1, \ldots, n-1$. Аналогично (3) и (2), из этого следует, что $\left(h+x^{\prime}\right)\left(t+t^{\prime}\right)$ принадлежит тому же смежному классу, что и $\left(h+x_{1}\right)\left(t+y_{1}\right)$. В силу произвольности выбора $x^{\prime} \in G F(q)$ этому смежному классу принадлежат по крайней мере $q$ элементов.

Теперь докажем, что элементы $[h] \cdot[t]$ попадают по крайней мере в $q$ различных классов. Зафиксируем элемент $x \in G F(q)$. Пусть для $y_{1}, y_{2} \in G F(q), y-1 \neq y_{2}$, произведения $(h+x)\left(t+y_{1}\right)$ и $(h+x)\left(t+y_{2}\right)$ принадлежат одному смежному классу. Равенства (3) при $x_{1}=x_{2}=x$ и $y_{1} \neq y_{2}$ могут выполняться, только если $h_{i}=0$ для всех $i=1, \ldots, n-1$, что противоречит условию $[h] \neq[0]$. Таким образом, произведения $(h+x)(t+y)$ при фиксированном $x \in G F(q)$ попадают в $q$ различных классов при различных $y \in G F(q)$.

Осталось доказать, что если некоторый смежный класс содержит по крайней мере два элемента из произведения $[h] \cdot[t]$, то еще $q-1$ различных смежных классов содержат по крайней мере два элемента (а значит, по крайней мере $q$ элементов) из этого произведения. Если для $x_{1}, x_{2}, y_{1}, y_{2}$ из $G F(q)$, где $x_{1} \neq x_{2}$, выполняются равенства (3), то, аналогично (4) и (5), для всех $i=1, \ldots, n-1$ выполняются равенства $h_{i} y_{1}^{\prime}+t_{i} x_{1}=h_{i} y_{2}^{\prime}+t_{i} x_{2}$ при $y_{2}^{\prime}=c\left(x_{1}-x_{2}\right)+y_{1}^{\prime}$, то есть произведения $\left(h+x_{1}\right)\left(t+y_{1}^{\prime}\right)$ и $\left(h+x_{2}\right)\left(t+y_{2}^{\prime}\right)$ принадлежат одному смежному классу. По доказанному ранее, эти классы различны при различных $y_{1}^{\prime} \in G F(q)$.

Обобщая полученные результаты, получаем, что если есть смежный класс, содержащий два элемента из $[h] \cdot[t]$, то в нем содержится не менее $q$ элементов этого множества, 
кроме того, существует еще $q-1$ различных смежных классов, каждый из которых содержит не менее $q$ элементов этого множества. Число элементов в $[h] \cdot[t]$ равно $q^{2}$, поэтому каждый из $q$ указанных смежных классов содержит ровно $q$ элементов этого произведения. Если не существует смежного класса, содержащего больше одного элемента из множества $[h] \cdot[t]$, то все $q^{2}$ элементов из $[h] \cdot[t]$ содержатся в различных смежных классах. Предложение доказано.

Следствие 2. В случае квадратичного расширения произведение $[h] \cdot[t]$, где $[h],[t] \in$ $G F\left(q^{2}\right) / G F(q) u[h] \neq[t]$, содержит q элементов (не обязательно различных) из каждого смежного класса.

Замечание 1. В дальнейшем в качестве представителя, задающего смежный класс, будем выбирать элемент, младшая координата которого в базисе $\left\{1, \alpha, \alpha^{2}, \ldots, \alpha^{n-1}\right\}$ равна нулю, то есть для смежного класса $[h]$ справедливо равенство $h=\sum_{i=1}^{n-1} h_{i} \alpha^{i}$, где $h_{i} \in G F(q)$.

Замечание 2. Из доказательства предложения 3 следует, что элементы произведения смежных классов $[h]$ и $[t]$ попадает ровно в $q$ смежных классов тогда и только тогда, когда существует $c \in G F(q)$ такое, что $t=h c$.

В случае, когда элементы произведения двух смежных классов попадают ровно в $q$ смежных классов, эти элементы не обязательно различны. Более точное описание элементов, попадающих в один смежный класс, дает следующее утверждение.

Предложение 4. Пусть во введенных выше обозначениях выполняется равенство $t=h c$, где с $\in G F(q)$. Тогда справедливы следующие утверждения.

(1) Все элементы произведения [h] · [0], попадающие в смежный класс [0], равны нулю. Все остальные элементы этого произведения различны.

(2) При $[t] \neq[0]$ и нечетном q элементы произведения $[h] \cdot[t]$, попадаюшие в один смежный класс, принимают $(q+1) / 2$ значений. При этом $(q-1) / 2$ из них встречаются в этом произведении по два раза.

(3) При $[t] \neq[0]$ и четном q существует один смежный класс такой, что все элементь произведения $[h] \cdot[t]$, попадающие в него, различны, а элементы, попадающие в остальные смежные классы, разбиваются на пары совпадающих.

Доказательство. Рассмотрим два элемента $\left(h+x_{1}\right)\left(t+y_{1}\right)$ и $\left(h+x_{2}\right)(t+y-2)$ произведения $[h] \cdot[t]$, где $x_{1} \neq x_{2}$, попадающие в один смежный класс. Найдем условия, при которых эти элементы совпадают. Как показано при доказательстве предложения 3 , $y_{2}=c\left(x_{1}-x_{2}\right)+y_{1}$, где $c-$ та же константа, которая связывает $t$ и $h$. Раскрыв скобки, приведя подобные члены и сократив на $x_{1}-x_{2}$, получаем, что равенство

$$
\left(h+x_{1}\right)\left(h c+y_{1}\right)=\left(h+x_{2}\right)\left(h c+c\left(x_{1}-x_{2}\right)+y_{1}\right)
$$

равносильно равенству

$$
x_{2} c=y_{1} .
$$

Рассмотрим сначала случай $c=0$. В этом случае $t=0$. Если $y_{1}=0$, то (7) выполняется для любого $x_{2} \in G F(q)$, то есть все $q$ значений, попавших в данный смежный класс, будут совпадать. Это значение равно 0 , поскольку $t+y_{1}=0$. Если $y_{1} \neq 0$, то (7) не 
выполняется ни для какого $x_{2} \in G F(q)$, то есть все $q$ значений, попадающих в каждый класс, будут различны. Это доказывает первую часть.

Если $c \neq 0$, то (7) дает решение уравнения (6), отличное от $x_{1}$, если $y_{1} \neq x_{1} c$, то есть значение произведения $(h+x)(t+x c)$ для любого $x \in G F(q)$ встречается в $[h] \cdot[t]$ один раз. Осталось рассмотреть распределение по смежным классам таких произведений. Произведения $\left(h+x_{1}\right)\left(t+x_{1} c\right)$ и $\left(h+x_{2}\right)\left(t+x_{2} c\right)$ попадают в один смежный класс тогда и только тогда, когда $x_{2} c=c\left(x_{1}-x_{2}\right)+x_{1} c$. Последнее равенство равносильно условию $2 x_{2} c=2 x_{1} c$. Поскольку $c \neq 0$, это условие при $x_{2} \neq x_{1}$ не выполняется в поле нечетной характеристики, а в поле четной характеристики выполняется для любого $x_{2} \in G F(q)$. Это завершает доказательство предложения.

\section{5. Заключение}

Показано, что сложение в конечном поле хорошо перемешивает смежные классы по мультипликативной группе любого подполя и наоборот. Это снижает эффективность использования гомоморфизмов для шифров, построенных с использованием операций в конечном поле. Также показано, что операция сложения в конечном поле равномерно перемешивает смежные классы по мультипликативной подгруппе, порядок которой близок к значению квадратного корня из числа элементов в поле. Для утверждения неэффективности использования гомоморфизмов в подгруппы такого порядка необходимо доказать аналогичный результат для операции сложения.

\section{Список литературы}

1. Шеннон К., Теория связи в секретных системах. В сб.: Работы по теории информации и кибернетике. Иностранная литература, Москва, 1963.

2. Горчинский Ю. Н., О гомоморфизмах многоосновных универсальных алгебр в связи с криптографическими применениями. Труды по дискретной математике (1997) 1, 67-84.

3. Горчинский Ю. Н., Стохастические алгебры. Труды по дискретной математике (1998) 2, 55-87.

Статья поступила 16.11.2010. 